\title{
Terahertz Luminescence and Absorption under Impurity Breakdown in Quantum Wells and Strained Semiconductor Layers
}

\author{
L.E. Vorobjev ${ }^{a}$, D.A. Firsov ${ }^{a}$, V.A. Shalygin ${ }^{a}$, \\ V.Yu. PAnevin ${ }^{a, *}$, A.N. Sofronov ${ }^{a}$, V.M. Ustinov ${ }^{b}$, \\ A.E. Zhukov ${ }^{b}$ A.Yu. Egorov ${ }^{b}$, A.V. Andrianov ${ }^{b}$, \\ A.O. Zakhar'in ${ }^{b}$, S.D. GanicheV ${ }^{c}$, S.N. Danilov $^{c}$ \\ AND D.V. KOZLOV ${ }^{d}$ \\ ${ }^{a}$ St. Petersburg State Polytechnic University \\ 29 Polytechnicheskaya st., 195251 St. Petersburg, Russia \\ ${ }^{b}$ Ioffe Physico-Technical Institute, 194021 St. Petersburg, Russia \\ ${ }^{c}$ University of Regensburg, 93040 Regensburg, Germany \\ ${ }^{d}$ Institute for Physics of Microstructures \\ 603950 Nizhny Novgorod, Russia
}

We present the results of $\mathrm{THz}$ luminescence investigations in structures with Si-doped quantum wells and Be-doped GaAsN layers under strong lateral electric field. The peculiar property of these structures is the presence of resonant impurity states which arise due to dimensional quantization in quantum wells and due to built-in strain in GaAsN epilayers. The experimentally obtained $\mathrm{THz}$ emission spectra consist of the lines attributed to intra-center electron transitions between resonant and localized impurity states and to the electron transitions involving the subband states. Absorption of $\mathrm{THz}$ radiation and its temperature dependence was also studied in structure with tunnel-coupled quantum wells at equilibrium conditions and under electric field.

PACS numbers: 68.65.Fg, 71.55.-i, 71.55.Eq, 73.21.Fg, 73.61.Ey, 78.55.Cr, 78.66.-w, 78.67.De

\section{Introduction}

The lack of semiconductor based small and robust sources of terahertz radiation is an important problem. A promising system for the development of semi-

*corresponding author; e-mail: pvyu@rphf.spbstu.ru 
conductor based $\mathrm{THz}$ emitters makes use of impurity states including resonant states in semiconductor micro- and nanostructures. Here we report on the observation of spontaneous emission of terahertz radiation in structures with Si-doped GaAs/AlGaAs quantum wells (QWs) and Be-doped GaAsN epilayers subjected to a lateral electric field. The GaAsN/AlGaAs structures have attracted considerable attention due to their remarkable properties offering promising possibilities to tailor the band gap, lattice constant, built-in stress and band alignment in practical photonic devices. They also allow to fabricate the high $Q$-factor cavity by cleaving along basal planes. These factors make GaAsN/AlGaAs micro- and nanostructures especially suitable for designing the $\mathrm{THz}$ lasers. We present the results of calculation of the acceptor energy levels and demonstrate the emission mechanism involving the resonant impurity states in these structures. In addition, we measured terahertz absorption under equilibrium and at the electron heating in electric field in tunnel-coupled GaAs/AlGaAs QWs.

\section{Samples and experimental technique}

The structures were grown by MBE on (001) semi-insulating GaAs substrates. Sample with rectangular GaAs/AlGaAs QWs comprised 200 periods of $30 \mathrm{~nm}$ thick GaAs layers separated by $7 \mathrm{~nm} \mathrm{Al}_{0.3} \mathrm{Ga}_{0.7} \mathrm{As}$ barriers. Central part (10 nm thick) of each QW was doped by silicon up to $1 \times 10^{17} \mathrm{~cm}^{-3}$. The second sample comprised 10 periods of $0.1 \mu \mathrm{m}$ thick $\mathrm{GaAs}_{0.982} \mathrm{~N}_{0.018}$ Bedoped (up to $2 \times 10^{17} \mathrm{~cm}^{-3}$ ) layers separated by $0.5 \mu \mathrm{m}$ thick GaAs layers. Built-in compressive strain in that sample develops along the growth direction. The deformation splitting of hh and lh subbands of the valence band in the GaAsN layers as determined from the interband photoluminescence measurements reaches $21 \mathrm{meV}$. The wafers were cleaved into the lateral dimensions of $4.5 \times 4.5 \mathrm{~mm}^{2}$ for QWs and of $4 \times 4.9 \mathrm{~mm}^{2}$ for epilayers sample. The third structure used for absorption measurements, comprised 100 periods of Si-doped (up to $5 \times 10^{17} \mathrm{~cm}^{-3}$ ) pairs of tunnel-coupled QWs. One period of structure consisted of GaAs:Si $/ \mathrm{Al}_{0.38} \mathrm{Ga}_{0.62} \mathrm{As} / \mathrm{GaAs}: \mathrm{Si} / \mathrm{Al}_{0.38} \mathrm{Ga}_{0.62}$ As layers with corresponding layer thicknesses 6.8/1.5/4.8/12 nm. Measurements were made in $45^{\circ}$-prism sample geometry. Contacts were produced by fusing indium.

The integrated $\mathrm{THz}$ electroluminescence (EL) intensity was measured by Ge:Ga detector in the spectral range of $60-110 \mu \mathrm{m}$ at $4.2 \mathrm{~K}$. Spectra of luminescence were recorded with spectral resolution of $0.6 \mathrm{meV}$ using pump-out Fourier spectrometer operating in step scan mode described elsewhere [1]. A liquid helium cooled silicon bolometer equipped with band pass optical filter to provide operation in the spectral range of $5-350 \mathrm{~cm}^{-1}$ served as the detector of $\mathrm{THz}$ radiation. The sample was fed with the trains of four pulses; each pulse in the train was $10 \mu \mathrm{s}$ long with $1.74 \mathrm{~ms}$ time interval. The duty cycle of the train was $50 \%$ at the $75 \mathrm{~Hz}$ frequency.

Molecular cw laser operating at radiation wavelength of $118 \mu \mathrm{m}$ (photon energy is $10.5 \mathrm{meV}$ ) and output power of $10 \mathrm{~mW}$ was used in absorption experiments. 


\section{Results and discussion}

The energy spectrum of shallow acceptors in strained GaAsN layers has been calculated using the envelope function approximation. The acceptor Hamiltonian used is $4 \times 4$ matrix operator including the Luttinger Hamiltonian, the deformation term, and the Coulomb potential. Similarly to Ref. [2], we have used a cylindrical approach, considering the Hamiltonian to be invariant under rotation about the direction of the deformation. We used the energy spectrum of Si-doped QW structure calculated previously for a similar structure [3].

Measurements of $j-V$ characteristics at $4.2 \mathrm{~K}$ showed that the processes of impurity impact ionization are significant at electric fields exceeding $200 \mathrm{~V} / \mathrm{cm}$ for the $p$-GaAsN/GaAs structure and about $30 \mathrm{~V} / \mathrm{cm}$ for $n$-GaAs/AlGaAs QWs. The sharp rise of $\mathrm{THz}$ EL starts since impurity impact ionization begins and correlates with the considerable rise of conductivity. The measured spectra of $\mathrm{THz}$ radiation are shown in Fig. 1.
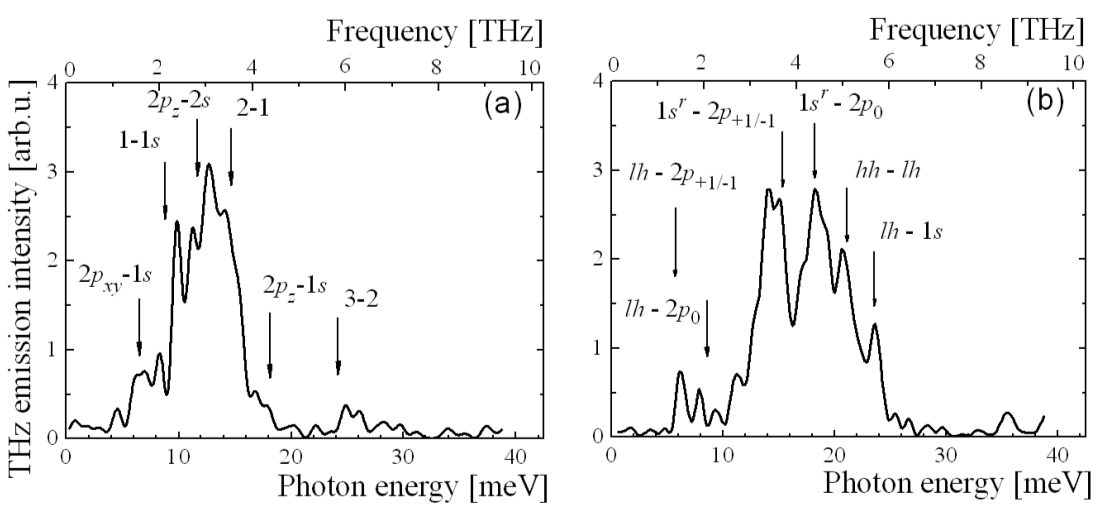

Fig. 1. Fourier spectra of EL for $n$-GaAs/AlGaAs QWs at $E=34 \mathrm{~V} / \mathrm{cm}$ (a) and for $p$-GaAsN/GaAs structure at $E=1600 \mathrm{~V} / \mathrm{cm}$ (b). $T=10 \mathrm{~K}$. Emission is registered from the sample surface. Arrows correspond to calculated transition energies.

The emission mechanism can be the following: at low temperature, the most of impurities are at the ground state. Free carriers generated by impurity ionization in external electric field are heated by the field and can be captured by the resonant state or scatter to the second subband. As a result, emission of terahertz radiation due to both the intersubband electron transitions and the transitions involving resonant and localized impurity states gets possible. The spectral position of emission lines for QW sample indicated that the emission is related to electron transitions between subbands $2-1$, between resonant and localized impurity states $2 p_{z}-2 s, 2 p_{z}-1 s$, and also to transitions $2 p_{x y}-1 s$ and $1-1 \mathrm{~s}$. For GaAsN sample the optical transitions of holes from the resonant acceptor level $1 s^{\mathrm{r}}$ to localized levels $2 p_{ \pm 1}$ and $2 p_{0}$ make the major contribution to the observed spectrum. The 
contributions of intersubband transitions of holes hh-lh and the band-acceptor level transitions $\mathrm{lh}-2 p_{ \pm 1}, \mathrm{lh}-2 p_{0}, \mathrm{lh}-1 s$ are less significant.

Absorption of polarized terahertz radiation was studied in the third sample applying radiation with photon energy of $10.5 \mathrm{meV}$. For that photon energy absorption is caused by indirect intrasubband (Drude-like) electron transitions with scattering by phonons or impurities. The obtained temperature dependence of equilibrium absorption and dependence of absorption modulation at lateral electric field are congruent as expected. At low temperature the free electron absorption is due to the electron scattering by ionized impurities. The decrease in the absorption with rising the temperature at low field strength is attributed to the heating of the electron gas by the electric field which reduces the probability of impurity scattering. The increase in radiation absorption detected at high fields (as well as at high temperatures) is caused by the change of scattering mechanism with further increase in the electron temperature from the scattering by ionized impurities to the scattering by optical phonons.

\section{Acknowledgments}

The work was supported by the RFBR, DFG, Russian President's program for support of young candidates of science, the Russian Science Support Foundation and the Russian Ministry of Education and Science.

\section{References}

[1] A.V. Andrianov, A.O. Zakhar'in, I.N. Yassievich, N.N. Zinov'ev, JETP Lett. 79, 365 (2004).

[2] V.Ya. Aleshkin, B.A. Andreev, V.I. Gavrilenko, I.V. Erofeeva, D.V. Kozlov, O.A. Kuznetsov, Nanotechol. 11, 348 (2000).

[3] M. Helm, F. M. Peeters, F. DeRosa, E. Colas, J.P. Harbison, L.T. Florez. Phys. Rev. B 43, 13983 (1991). 